\title{
RADIAL LIMITS AND GROWTHS OF FRACTIONAL CAUCHY TRANSFORMS
}

\author{
T. H. MACGREGOR
}

Department of Mathematics and Statistics, State University of New York at Albany

Albany, New York 12222, U.S.A.

1. Let $\Delta=\{z:|z|<1\}$ and $\Gamma=\{z:|z|=1\}$, and let $\mathcal{M}$ denote the set of complex-valued Borel measures on $\Gamma$. A family denoted $\mathcal{F}_{\alpha}$ of functions analytic in $\Delta$ is defined for each $\alpha \geq 0$ as follows. For $\alpha>0, f \in \mathcal{F}_{\alpha}$ provided that there exists $\mu \in \mathcal{M}$ such that

$$
f(z)=\int_{\Gamma} \frac{1}{(1-\bar{\zeta} z)^{\alpha}} d \mu(\zeta)
$$

for $|z|<1$. Here and elsewhere each logarithm is the principal branch. Also, $f \in \mathcal{F}_{0}$ provided that there exists $\mu \in \mathcal{M}$ such that

$$
f(z)=f(0)+\int_{\Gamma} \log \frac{1}{1-\bar{\zeta} z} d \mu(\zeta)
$$

for $|z|<1 . \mathcal{F}_{\alpha}$ is a Banach space with respect to the norm defined by $\|f\|_{\mathcal{F}_{\alpha}}=$ inf $\|\mu\|$ where $\mu$ varies over all measures in $\mathcal{M}$ for which (1) or (2) holds and where $\|\mu\|$ is the total variation norm of $\mu$.

The spaces $\mathcal{F}_{\alpha}$ have been studied in a number of recent papers and earlier work was extensively done corresponding to the case $\alpha=1$. This paper gives a survey of some recent results about $\mathcal{F}_{\alpha}$ which focus on questions about radial limits. We will summarize work contained in [3], [4] and [5]. This material was presented in a talk by the author at the Complex Analysis Semester held at the Banach Center in Warsaw during the fall, 1992. The author wishes to thank the organizers for their invitation to participate in that conference and for their hospitality, especially to Professor Jan Krzyż.

1991 Mathematics Subject Classification: 30D99, 30E20, 31A20.

The paper is in final form and no version of it will be published elsewhere. 
2. The following result was proved in [3; see Theorem 1] and is one of several relations known between $\mathcal{F}_{\alpha}$ and other spaces.

Theorem 1. If $0<\alpha \leq 1$ then $\mathcal{F}_{\alpha} \subset H^{p}$ for $0<p<1 / \alpha$. Also $\mathcal{F}_{0} \subset H^{p}$ for all $p>0$.

Theorem 1 and classical results about $H^{p}$ spaces [2] imply the following fact about radial limits of functions in $\mathcal{F}_{\alpha}$ : if $f \in \mathcal{F}_{\alpha}$ and $0 \leq \alpha \leq 1$ then $f\left(e^{i \theta}\right)$ exists for almost all $\theta$ in $[-\pi, \pi]$. Here $f\left(e^{i \theta}\right)$ is defined by $f\left(e^{i \theta}\right)=\lim _{r \rightarrow 1-} f\left(r e^{i \theta}\right)$ when this limit exists. We also recall the idea of a nontangential limit. Let $S(\theta, \gamma)$ denote the Stolz angle in $\Delta$ having vertex $e^{i \theta}$ and opening $\gamma$ where $0<\gamma<\pi$. Then $f$ has a nontangential limit at $e^{i \theta}$ provided that $\lim _{z \rightarrow e^{i \theta}, z \in S(\theta, \gamma)} f(z)$ exists for every $\gamma(0<\gamma<\pi)$.

Later we describe a sufficient condition for a function in $\mathcal{F}_{\alpha}$ to have a radial limit in a given direction. This helps to show that if $f \in \mathcal{F}_{\alpha}$ and $0 \leq \alpha<1$ then $f\left(e^{i \theta}\right)$ exists off certain exceptional sets depending on $\alpha$ and which are generally thinner than sets of measure zero. This involves the notion of capacity. Also we describe what can happen when $\alpha>1$. At this point we note that to each $\alpha>1$ there exists a function $f \in \mathcal{F}_{\alpha}$ which belongs to no $H^{p}$ space; in fact, there exists such $f$ for which $\varlimsup_{r \rightarrow 1-}\left|f\left(r e^{i \theta}\right)\right|=\infty$ for all $\theta$ in $[-\pi, \pi]$.

Let $\tilde{\mathcal{M}}$ denote the subset of $\mathcal{M}$ consisting of probability measures, and let $\tilde{\mathcal{F}}_{\alpha}$ denote the subset of $\mathcal{F}_{\alpha}$ consisting of functions for which (1) or (2) holds for some $\mu \in \tilde{\mathcal{M}}$.

The next theorem gives a connection between the existence of radial limits and that of nontangential limits.

THEOREM 2. If $f \in \tilde{\mathcal{F}}_{\alpha}$ for some $\alpha, 0 \leq \alpha \leq 1$ and $f\left(e^{i \theta}\right)$ exists, then $f$ has a nontangential limit at $e^{i \theta}$.

The proof of Theorem 2 is given in [4; see Theorem 2]. The argument consists of noting that the kernels $F_{\alpha}(z)=1 /(1-z)^{\alpha}$ (when $\left.0<\alpha \leq 1\right)$ and $F_{0}(z)=$ $\log (1 /(1-z))$ are convex, univalent mappings of $\Delta$. This implies that each $f \in \tilde{\mathcal{F}}_{\alpha}$ is subordinate to $F_{\alpha}$. Thus $f=F \circ \varphi$ for some function $\varphi$ which is analytic in $\Delta$ and satisfies $|\varphi(z)|<1$ and $\varphi(0)=0$. The existence of $f\left(e^{i \theta}\right)$ implies the existence of $\varphi\left(e^{i \theta}\right)$. By a classical result about bounded analytic functions [2,p.6], $\varphi$ has a nontangential limit at $e^{i \theta}$. Therefore $f$ has a nontangential limit at $e^{i \theta}$.

3. Equation (1) is equivalent to

$$
f(z)=\int_{-\pi}^{\pi} \frac{1}{\left(1-e^{-i t} z\right)^{\alpha}} d g(t)
$$

where $g$ is a complex-valued function of bounded variation on $[-\pi, \pi]$. Likewise, for such a $g,(2)$ can be rewritten

$$
f(z)=f(0)+\int_{-\pi}^{\pi} \log \left(\frac{1}{1-e^{-i t} z}\right) d g(t) .
$$


THEOREM 3. Suppose that the function $f$ is defined by (3) or by (4) where $g$ is of bounded variation on $[-\pi, \pi]$. If

$$
\int_{-\pi}^{\pi} \frac{|g(\theta+t)-g(\theta)|}{|t|^{\alpha+1}} d t<\infty
$$

for some $\theta$ in $[-\pi, \pi]$, then $f\left(e^{i \theta}\right)$ exists.

Theorem 3 is proved in [4]. The argument depends on an integration by parts and an application of the Lebesgue dominated convergence theorem. This result shows what local behavior of $g$ near $\theta$ implies $f\left(e^{i \theta}\right)$ exists. As pointed out later, this implies a global result about radial limits with suitable exceptional sets for functions in $\mathcal{F}_{\alpha}$ in the case $0 \leq \alpha<1$.

4. Next we describe a few facts about zero $\alpha$-capacity. Reference [1] is a source for information about capacity.

For $0<\alpha<1$ let

$$
P_{\alpha}(\theta)=\frac{1}{\left|\sin \frac{1}{2} \theta\right|^{\alpha}} \quad(|\theta| \leq \pi),
$$

and let

$$
P_{0}(\theta)=\log \frac{1}{\left|\sin \frac{1}{2} \theta\right|} \quad(|\theta| \leq \pi) .
$$

A nonempty Borel set $E \subset[-\pi, \pi]$ is said to have positive $\alpha$-capacity provided that there exists a probability measure $\mu$ supported on $E$ such that

$$
\sup _{\theta} \int_{-\pi}^{\pi} P_{\alpha}(\theta-t) d \mu(t)<\infty .
$$

Otherwise $E$ is said to have zero $\alpha$-capacity and we write $C_{\alpha}(E)=0$. Intuitively, $E$ has zero $\alpha$-capacity when $E$ is so thin that no probability measure can be distributed on $E$ which "cancels out" the singularity of $P_{\alpha}$. The general idea of capacity when $\alpha=0$ corresponds to the notion of logarithmic capacity. Zero $\alpha$-capacity is a useful way to describe the fineness of exceptional sets for problems about $\mathcal{F}_{\alpha}$.

A few facts about zero $\alpha$-capacity are listed in the next theorem. Each assertion is easy to prove.

Theorem 4. (a) If $E \subset F$ and $C_{\alpha}(F)=0$ then $C_{\alpha}(E)=0$.

(b) If $C_{\alpha}(E)=0$ and $\beta>\alpha$ then $C_{\beta}(E)=0$.

(c) If $C_{\alpha}(E)=0$ and $C_{\alpha}(F)=0$ then $C_{\alpha}(E \cup F)=0$.

(d) If $E$ is finite or countable then $C_{\alpha}(E)=0$ for all $\alpha$.

(e) If $C_{\alpha}(E)=0$ for some $\alpha$ then the Lebesgue measure of $E$ is zero.

The following result was proved by J. B. Twomey in [6] 
THEOREM 5. Let $g$ be a real-valued nondecreasing function on $[-\pi, \pi]$ and let $0 \leq \alpha<1$. Then

$$
\int_{0}^{\pi} \frac{g(\theta+t)-g(\theta-t)}{t^{\alpha+1}} d t<\infty
$$

for all $\theta$ in $[-\pi, \pi]$ except possibly for a set having zero $\alpha$-capacity.

The argument for Theorem 5 depends on first showing that if

$$
Q_{\alpha}(\theta) \equiv \int_{-\pi}^{\pi} P_{\alpha}(\theta-t) d g(t) \text { and } E \equiv\left\{\theta: Q_{\alpha}(\theta)=\infty\right\}
$$

then $C_{\alpha}(E)=0$. Next it is shown that (9) holds for each $\theta$ for which $Q_{\alpha}(\theta)<\infty$.

TheOREM 6. If $f \in \mathcal{F}_{\alpha}$ for some $\alpha$ where $0 \leq \alpha<1$, then $f$ has a nontangential limit at $e^{i \theta}$ for all $\theta$ in $[-\pi, \pi]$ except possibly for a set having zero $\alpha$-capacity.

The proof of Theorem 6 given in [4] is as follows. By appealing to the Jordan decomposition theorem and part (c) of Theorem 4 we see that it suffices to assume that $f \in \tilde{\mathcal{F}}_{\alpha}$. Theorem 2 implies we need only treat radial limits. Because $f \in \tilde{\mathcal{F}}_{\alpha}$ we have (3) or (4) where $g$ is nondecreasing (and $g(\pi)-g(-\pi)=1$ ). It is easy to see that (9) implies (5). Hence Theorems 5 and 3 complete the argument for Theorem 6 .

5. When $\alpha>1$ the existence of a function of $f \in \mathcal{F}_{\alpha}$ such that $\overline{\lim }_{r \rightarrow 1-}\left|f\left(r e^{i \theta}\right)\right|$ $=\infty$ for all $\theta$ on $[-\pi, \pi]$ is only one consequence of a study about the growth of $|f|$ off suitable exceptional sets. This study makes sense for all $\alpha \geq 0$.

First of all, we note that if $f \in \mathcal{F}_{\alpha}$ and $\alpha>0$ then (1) implies that $|f(z)| \leq$ $\|\mu\| /(1-r)^{\alpha}$ for $|z| \leq r$. In other words, $|f(z)|=\mathcal{O}\left[1 /(1-r)^{\alpha}\right]$ for $0<r<1$. This maximal growth is attained by the function represented by point mass, say at $e^{i \theta}$, and where the radial growth is in the direction of $\theta$. The next two results show that certain smaller radial growths can be associated with suitable exceptional sets.

TheOREM 7. If $f \in \mathcal{F}_{\alpha}$ and $\alpha>0$, then $\lim _{r \rightarrow 1-}(1-r)^{\alpha} f\left(r e^{i \theta}\right)=0$ for all $\theta$ in $[-\pi, \pi]$ except possibly for a finite or countable set.

TheOREM 8. If $f \in \mathcal{F}_{\alpha}$ and $\alpha>1$, then $\lim _{r \rightarrow 1-}(1-r)^{\alpha-1} f\left(r e^{i \theta}\right)=0$ for almost all $\theta$ in $[-\pi, \pi]$.

The proof of Theorem 7 given in [4] is quite simple. It depends on the Lebesgue bounded convergence theorem and the fact that for a Borel measure $\mu \neq 0$ the set $E \subset[-\pi, \pi]$ for which $\mu\left(\left\{e^{i \theta}\right\}\right) \neq 0$ is either empty, finite or countable.

A proof of Theorem 8 was first given in [3]. A second proof was obtained in [4]. The second argument relies on the following local result. Theorem 8 is an immediate consequence of this result and the fact that a function of bounded variation is differentiable almost everywhere. 
TheOREM 9. Suppose that $g$ is a function of bounded variation on $[-\pi, \pi]$ and $\alpha>1$, and let $f$ be defined by (3). If $g$ is differentiable at $\theta$ then $\lim _{r \rightarrow 1-}(1-r)^{\alpha-1} f\left(r e^{i \theta}\right)=0$.

There are additional conclusions obtained in [4] and [5] showing how the local behavior of the function $g$ near $\theta$ or the measure $\mu$ near $e^{i \theta}$ affects the radial growth of $f$ in the direction $e^{i \theta}$. In particular, in [5] this leads to the following theorem concerning growths which are intermediate to those described in Theorems 7 and 8 .

TheOREM 10. Suppose that $f \in \mathcal{F}_{\alpha}$ for some $\alpha>0,0<\beta<1$ and $\beta<\alpha$. Then $\lim _{r \rightarrow 1-}(1-r)^{\alpha-\beta} f\left(r e^{i \theta}\right)=0$ for all $\theta$ in $[-\pi, \pi]$ except possibly for a set having zero $\beta$-capacity.

Several of the results quoted above which are stated in terms of radial limits were proved in a more general form for suitable nontangential limits. Also there are results which correspond to the cases where $\alpha=0$ and, in Theorem 10, where $\beta=0$.

6. The next theorem describes how large $\min _{|z|=r}|f(z)|$ can be for sequences $\left\{r_{n}\right\}$ where $r_{n} \rightarrow 1, f \in \mathcal{F}_{\alpha}$ and $\alpha>1$.

THEOREM 11. Let $\alpha>1$ and let $\varepsilon$ be a real-valued nonincreasing function on $(0,1)$ such that $\lim _{r \rightarrow 1-} \varepsilon(r)=0$. Then there exists $f \in \mathcal{F}_{a}$ such that

$$
\varlimsup_{r \rightarrow 1-}\left\{\frac{(1-r)^{\alpha-1}}{\varepsilon(r)} \min _{|z|=r}|f(z)|\right\}=\infty .
$$

Theorem 11 asserts that for any "growth" less than $o\left[\frac{1}{(1-r)^{\alpha-1}}\right]$ there is $f \in \mathcal{F}_{\alpha}$ such that the exceptional set for that growth is all of $[-\pi, \pi]$. In particular, for suitable choices of $\varepsilon$, (10) implies that $\varlimsup_{r \rightarrow 1-}\left|f\left(r e^{i \theta}\right)\right|=\infty$ for all $\theta$.

We outline the proof of Theorem 11 given in [3]. Let the sequence $\left\{A_{n}(\alpha)\right\}$ be defined by $1 /(1-z)^{\alpha}=\sum_{n=0}^{\infty} A_{n}(\alpha) z^{n}$. It is easy to see that $f \in \mathcal{F}_{\alpha}$ if and only if $g \in \mathcal{F}_{1}$ where $f$ and $g$ are related by $f(z)=\sum_{n=0}^{\infty} a_{n} z^{n}, g(z)=\sum_{n=0}^{\infty} b_{n} z^{n}$ and $b_{n}=a_{n} / A_{n}(\alpha)$ for $n=0,1, \ldots$ Let $\left\{\lambda_{n}\right\}$ be an increasing sequence of positive integers and let $g(z)=\sum_{n=1}^{\infty} \frac{1}{n^{2}} z^{\lambda_{n}}$ and $f(z)=\sum_{n=1}^{\infty} \frac{1}{n^{2}} A_{\lambda_{n}}(\alpha) z^{\lambda_{n}}$ for $|z|<1$. Clearly $g$ is bounded in $\Delta$ and hence Cauchy's formula gives $g \in \mathcal{F}_{1}$. Therefore $f \in \mathcal{F}_{\alpha}$. Let $\alpha$ and $\varepsilon$ satisfy the assumptions in Theorem 11. Then $\left\{\lambda_{n}\right\}$ can be chosen so that

$$
\lambda_{k+1} \geq[k+1]^{3 /(\alpha-1)} \lambda_{k}
$$

and

$$
\varepsilon\left(1-1 / \lambda_{k}\right) \leq 1 / k^{2}
$$

for $k=1,2, \ldots$ The existence of $\left\{\lambda_{n}\right\}$ which satisfies (11) is easy to verify inductively and (12) is possible to satisfy simultaneously since $\varepsilon(r) \rightarrow 0$ as $r \rightarrow 1-$. 
For $k=1,2, \ldots$ let $r_{k}=1-1 / \lambda_{k}$

$$
P_{k}(z)=\sum_{n=1}^{k-1} \frac{1}{n^{2}} A_{\lambda_{n}}(\alpha) z^{\lambda_{n}}, \quad Q_{k}(z)=\frac{1}{k^{2}} A_{\lambda_{k}}(\alpha) z^{\lambda_{k}}
$$

and

$$
R_{k}(z)=\sum_{n=k+1}^{\infty} \frac{1}{n^{2}} A_{\lambda_{n}}(\alpha) z^{\lambda_{n}}
$$

for $|z|<1$. Then $f(z)=P_{k}(z)+Q_{k}(z)+R_{k}(z)$. A technical but elementary argument shows that the condition (11) implies that $\left|P_{k}(z)\right| \leq \frac{1}{4}\left|Q_{k}(z)\right|$ and $\left|R_{k}(z)\right| \leq$ $\frac{1}{4}\left|Q_{k}(z)\right|$ for $|z|=r_{k}$ and for all large values of $k$. Hence $|f(z)| \geq \frac{1}{2}\left|Q_{k}(z)\right|$. Therefore, for $|z|=r_{k}$ and for all large $k$, we have $|f(z)| \geq \frac{1}{2} \frac{1}{k^{2}} A_{\lambda_{k}}(\alpha) r_{k}^{\lambda_{k}}$. There is a positive constant $C(\alpha)$ such that $A_{n}(\alpha) \geq C(\alpha) n^{\alpha-1}$ for $n \geq 1$. Hence (12) and $r_{k}^{\lambda_{k}} \rightarrow \frac{1}{e}$ imply that

$$
|f(z)| \geq \frac{C(\alpha)}{6} \frac{\varepsilon\left(r_{k}\right)}{\left(1-r_{k}\right)^{\alpha-1}}
$$

for $|z|=r_{k}$ and for all large $k$. This inequality implies the theorem.

\section{References}

[1] L. Carleson, Selected Problems on Exceptional Sets, Van Nostrand, New York, 1967.

[2] P. L. Duren, Theory of $H^{p}$ Spaces, Academic Press, New York, 1970.

[3] D. J. Hallenbeck and T. H. MacGregor, Growth and zero sets of Analytic families of Cauchy-Stieltjes integrals, J. Analyse Math. 61 (1993), 231-259.

[4] —, - Radial limits and radial growth of Cauchy-Stieltjes transforms, Complex Variables 21 (1993), 219-229.

[5] - - - Radial growth and exceptional sets for Cauchy-Stieltjes integrals, Proc. Edinburgh Math. Soc. 37 (1993), 73-89.

[6] J. B. Twomey, Tangential boundary behavior of the Cauchy integral, J. London Math. Soc. (2) 37 (1988), 447-454. 